\title{
AVALIAÇÃO DA ESCOLHA DOS TESTES DE SENSIBILIDADE PULPAR POR CLÍNICOS GERAIS DA CIDADE DE TAUBATÉ
}

\author{
EVALUATION OF THE CHOICE OF PULP SENSITIVITY TESTS BY \\ PRACTITIONERS OF TAUBATÉ CITY
}

\author{
João Marcelo Ferreira de Medeiros * \\ Claudia Auxiliadora Pinto * \\ Luiz Carlos Laureano da Rosa ${ }^{\cdots \cdot}$ \\ Sandra Marcia Habitante *.** \\ Edison Tibagy Dias Carvalho de Almeida …. \\ Nivaldo André Zollner *.....
}

\begin{abstract}
RESUMO
Introdução: A proposta desta pesquisa é avaliar os recursos complementares de que os clínicos gerais da cidade de Taubaté lançam mão na determinação da sensibilidade pulpar. Método: Foram entrevistados ao acaso 106 cirurgiões-dentistas da cidade de Taubaté valendo-se de um questionário elaborado pelo autor. Tais profissionais eram de diferentes épocas de graduação, portadores de especialidade ou não e de diversas faculdades de Odontologia. Os questionários apresentavam uma lista de testes com a seguinte pergunta: "Qual ou quais os recursos suplementares mais utilizados na avaliação da sensibilidade pulpar deque você lança mão durante o exame do paciente?". Os dados foram organizados de modo a facilitar sua subdivisão em teste pelo frio, calor, elétrico, cavidade, anestesia e outros, permitindo estabelecer percentuais de utilização destes em função do número de citações. Resultados: Como inferência estatística utilizou-se teste binomial para proporção, sendo o nível de significância de 5\%.Na comparação entre a proporção de clínicos gerais que utilizam guta-percha $(90,56 \%)$ e bastão de gelo $(77,35 \%)$ o teste apresentou pvalor $<0,05$ indicando que a proporção de clínicos gerais que usam guta-percha aquecida é maior do que aqueles clínicos que usam bastão de gelo. Conclusão: De posse dos resultados obtidos, concluiu-se que a guta-percha aquecida foi o recurso auxiliar preferido pelos clínicos gerais $(90,56 \%)$ seguido do bastão de gelo $(77,35 \%)$, gases refrigerantes $64,15 \%)$, jato de ar $(41,5 \%)$, teste de anestesia $(38,67 \%)$, teste de cavidade $(31,13 \%)$, água fria $(24,52 \%)$, brunidor aquecido $(4,71 \%)$, teste elétrico (1,88\%) e algodão embebido em álcool (0,94\%).
\end{abstract}

DESCRITORES: Diagnóstico • Teste da polpa dentária • Frio, Calor • Testes diagnósticos de rotina.

\section{ABSTRACT}

Introduction: The purpose of this study is to assess which methods that the general practitioners of the Taubaté city used to determine the pulpal sensitivity. Method: Were interviewed 106 randomly dentists of Taubate city making use of a questionnaire prepared by the author. These professionals were from different times of graduation, expert or not and from various dentistry colleges. The questionnaires presented a list of tests with the following question: which additional resources do you prefer during the evaluation of pulpal sensitivity during of the patient exam?". The data were organized to facilitate their subdivision: cold test, heat test, electric test, anesthesia test and cavity test, to establish rates of use of these depending on the number of citations. Results: Because of statistical inference was used the binomial proportion with the significance level of $5 \%$. The proportion of general practitioners using heat gutta-percha $(90.56 \%)$ and ice stick $(77.35 \%)$ tests presented pvalue $<0.05$ indicating that the proportion of general practitioners using heat gutta-percha is higher than those clinicians that using ice stick. Conclusion: It was concluded that heat gutta-percha were the test preferred by practitioners $(90.56 \%)$ followed by ice stick $(77.35 \%)$, refrigerants $(64.15 \%)$, air jet $(41.5 \%)$, test of anesthesia $(38.67 \%)$ test cavity $(31.13 \%)$, cold water $(24.52 \%)$, heated burnisher $(4.71 \%)$, electrical test $(1.88 \%)$ and alcohol swab (0.94\%).

DESCRIPTORS: Diagnosis $\bullet$ Dental pulp test $\bullet$ Cold temperature, hot temperature $\bullet$ Diagnostic tests, routine

* Professor Assistente Doutor da Disciplina de Endodontia do Departamento de Odontologia da Universidade de Taubaté. Professor do Programa de PósGraduação em Odontologia da Universidade de Taubaté E-mail: jmedeiros@unitau.br

** Professora Assistente Mestre da Disciplina de Endodontia do Departamento de Odontologia da Universidade de Taubaté E-mail: draclaudiapinto@uol. com.b

*** Professor Assistente Doutor da Disciplina de Bioestatística do Instituto Básico de Ciências Exatas da Universidade de Taubaté. Pesquisador do Núcleo de Pesquisas Econômico-Sociais da Universidade de Taubaté. Coordenador do NUPES. E-mail: laureanodarosa@gmail.com

**** Professora Assistente Doutora da Disciplina de Endodontia do Departamento de Odontologia da Universidade de Taubaté - UNITAU. Professora do Programa de Pós-Graduação em Odontologia da Universidade de Taubaté. Chefe de Departamento de Odontologia da Universidade de Taubaté E-mail: shabitante@uol.com.br

***** Professor Assistente Doutor da Disciplina de Clínica Integrada do Departamento de Odontologia da Universidade de Taubaté. E-mail: central_estagios@ unitau.br

****** Professor Doutor da Disciplina de Clínica Integrada do Departamento de Odontologia da Universidade de Taubaté. Chefe da Clínica Odontológica da Universidade de Taubaté. E-mail: andrezollner@yahoo.com.br 


\section{N T RO D UÇÃ O}

A determinação do diagnóstico endodôntico correto representa etapa inicial do tratamento endodôntico cuja responsabilidade pelo êxito ou fracasso depende do profissional e das inúmeras adversidades relacionadas não somente com alterações fisiopatológicas, mas também à manifestação do mecanismo da dor, fatores morfo- estruturais condicionados à idade do paciente, à intensidade e frequência do dano pulpar, entre outros fatores (Medeiros et al. ${ }^{1}$ 2007).

Assim, embora aparentemente difícil a elaboração do diagnóstico clínico da condição pulpar, resta a possibilidade da utilização de recursos semiotécnicos que, em parceria com a capacidade e habilidade do profissional, conduzem corretamente o tratamento.

Atestam Castagnola e Negro $^{2}$ (1972) que dentre os recursos utilizados no diagnóstico clínico do estado pulpar destacase o exame da sensibilidade pulpar, que acusa a presença de dor, identifica sua sede e diferencia dores de origem dentária ou não e quando o dente apresenta alterações de cor, dentes traumatizados, dentes com alterações estruturais, com lesões periodontais e/ou periapicais e quando de fato existe uma necessidade premente de uma avaliação acertada da condição pulpar.

Por outro lado, dentre os testes para avaliação da resposta pulpar, são reconhecidos os estímulos térmicos e elétrico, sendo este último recurso amplamente utilizado até a década de 80 . Ainda se discute quanto à sua confiabilidade, pois existe a possibilidade eminente de ocorrência de falsos resultados positivos ou negativos como, por exemplo, a presença de restaurações extensas, aparelhos ortodônticos, dentes traumatizados, dentes permanentes jovens ou com rizogênese incompleta ou ainda naqueles casos de atresia da câmara pulpar decorrente de alterações patológicas ou fisiológicas.

Portanto, os recursos mais simples e práticos, além daqueles de maior eficácia do ponto de vista clínico, são os métodos mais usados no dia-a-dia conhecidos como testes térmicos.
Desde a utilização de água resfriada (Jack ${ }^{3}$ 1899) passando pelo emprego de gelo acondicionado em recipientes de alumínio (Austin e Waggener ${ }^{4}$ 1941), e, até hoje, empregado em tubos de anestésicos vazios, os quais são preenchidos com água e congelados (Dachi et al. ${ }^{5}$ 1967, Pesce et al. ${ }^{6}$ 1985), diversos estudos vêm comprovando a eficácia do agente térmico pelo frio em provocar estímulos na unidade sensorial pulpar, tratando-se de método fácil e de rápida execução. No que respeita a qualificação do teste pelo frio em pressupor a vitalidade pulpar, convém elucidar ainda a possibilidade de aquilatar o grau de reversibilidade ou de irreversibilidade do processo inflamatório instalado na polpa.

É claro que a capacidade em se estabelecer um diagnóstico endodôntico correto diferencia o dentista prático do verdadeiro cirurgião-dentista, criando responsabilidade pelos sucessos e insucessos. Essa concepção aparentemente difícil do diagnóstico é minimizada pela possibilidade da utilização de recursos auxiliares que, em parceria com a capacidade e habilidade do profissional, conduzem correta e coerentemente o tratamento.

Mais ainda, importa relatar que o exercício do diagnóstico pulpar tem confirmado o valor do resfriamento como recurso complementar de exame durante a determinação do diagnóstico do estado pulpar, inclusive em diversas especialidades odontológicas (Aun et al. ${ }^{7}$ 1992; Aun et al. ${ }^{8}$ 1994; Pesce et al. ${ }^{9}$ 1997; Medeiros e Pesce $^{10}$ 1997; Medeiros e Pesce ${ }^{11}$ 1998; Medeiros et al. ${ }^{12}$ 2004; Medeiros et al. ${ }^{13}$ 2005; Medeiros et al. ${ }^{1}$ 2007; Haddad FiIho et al. ${ }^{14}$ 2009).

De outro modo não se justificam as limitações que o teste térmico com gelo decorrente de complicações de ordem anatômicas, fisiológicas ou histopatológicas. Por exemplo, situações de dentes com maior espessura de dentina ou com restauração extensa, de pacientes idosos ou muito jovens, ainda considerando as complicações de cunho psicológico. Episódios como esses dificultam e até mesmo impedem uma análise mais sensata da condição pulpar, o que gera insegurança sobre o caminho a ser instituído durante a
MEDEIROS JMF

PINTO CA

Rosa LCL

HABITANTE SM

ALMEIDA ETDC

ZOLLNER NA

AVALIAÇÃO DA

ESCOLHA DOS

TESTES DE

SENSIBILIDADE

PULPAR POR

CLÍNICOS GERAIS

DA CIDADE DE

TAUBATÉ

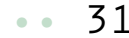

31

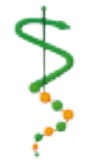

REVISTA DE

ODONTOLOGIA DA

UN I VERS I DADE

CidAdE DE São

PAULO

$2010 ; 22(1): 30-8$, JAN - ABR 
MEDEIROS JMF

PINTO CA

ROSA LCL

HABITANTE SM

ALMEIDA ETDC

ZOLLNER NA

AVALIAÇÃO DA

ESCOLHA DOS

TESTES DE

SENSIBILIDADE

PULPAR POR

CLINNICOS GERAIS

DA CIDADE DE

TAUBATÉ

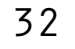

REVISTA DE

ODONTOLOGIA DA

UNI VERS I DADE

CIDADE DE SÃO

PAULO

$2010 ; 22(1): 30-8$,

$J A N-A B R$ terapia que se segue.

Procura-se utilizar recursos alternativos mais eficientes do que o teste térmico, com o gelo ou mesmo o teste elétrico preferindo-se substâncias dotadas de baixa temperatura, como as substâncias refrigerantes como tetrafluoroetano, propano/butano, neve carbônica e o cloreto de etila.

Esses métodos são dotados de alta capacidade refrigerante com temperaturas entre -46 e $-76^{\circ} \mathrm{C}$ e com capacidade de diminuir sua temperatura na fonte e nos meios os quais são transportados (Irala ${ }^{15}$ 2003, Pesce et al. ${ }^{16}$ 1995), de fácil manipulação, excelente aplicação e, em decorrência, com eficácia clínica comprovada (Pesce e Medeiros ${ }^{17}$ 1998) sem, inclusive, danificar a estrutura dentária (Barletta ${ }^{18}$ 1994).

Importa considerar, para fins diagnósticos, o uso dos testes pelo calor, principalmente a guta-percha aquecida, aplicada sobre o esmalte dentário. Esse tipo de teste sempre foi severamente criticado, fundamentalmente quanto à expectativa de produzir falsos resultados negativos, segundo atestam, aliados à dificuldade do controle da alta temperatura quando de sua aplicação, além de relatos de dor intensa (Cohen e Burns ${ }^{19}$ 2000).

Aliás, Nicholls ${ }^{20}$ (1984) assegura que dos recursos semiotécnicos à disposição dos clínicos para o estabelecimento do diagnóstico da condição pulpar, na maioria das vezes, poucos são necessários, mesmo que, em algumas ocasiões, muitos sejam empregados.

$\mathrm{Na}$ verdade um dos sintomas mais comuns de dor em dentes com sintomatologia álgica portadores de polpa dentária inflamada é a dor provocada pela estimulação térmica. Assim é que determinados tipos de dor de origem pulpar podem ser provocados ou aliviados pela aplicação de estímulos frios ou quentes. Por exemplo, nos estágios onde a polpa dentária apresenta-se com dor difusa e irradiada, as variações térmicas, repetidas vezes, auxiliam a localização, com precisão, da origem do dente causal (Lage-Marques e Antoniazzi ${ }^{21}$ 2002).

Deve-se incluir informação correta da manipulação desses recursos, pois, sua boa recomendação refletirá numa melhor otimização do diagnóstico e, por conseguinte, uma melhor maneira de conduzila, quando manifestações incomuns se apresentarem.

Convém lembrar que, além dos testes térmicos, tanto por resfriamento quanto por aquecimento, emprega-se também a corrente elétrica.

Relativamente a esta última, apesar de amplamente utilizada até a década de 80, muito se discute quanto à sua eficácia, uma vez que existe a possibilidade eminente da ocorrência de falsos resultados positivos e negativos. Por exemplo, presença de restaurações extensas, apareIhos ortodônticos, dentes traumatizados, dentes permanentes jovens ou com rizogênese incompleta ou ainda aqueles com atresia da câmara pulpar decorrente de alterações patológicas e fisiológicas (Cohen e Burns ${ }^{19}$ 2000).

Mas não é só. Situações existem onde se pode recorrer aos testes de anestesia e de cavidade embora se considere este último recurso invasivo, porém eficaz (Paiva e Antoniazzi22 1991).

Com vistas a isso, objetivou-se investigar, neste estudo, o método de escolha na detecção da sensibilidade pulpar de cirurgiões-dentistas da cidade de Taubaté.

\section{MATERIAL E MÉTODOS}

Foram entrevistados randomicamente 106 cirurgiões-dentistas da cidade de Taubaté, valendo-se de um questionário elaborado pelo autor. Cumpre observar que os entrevistados eram clínicos gerais pertencentes a diferentes épocas de graduação, alguns portadores de especialidade e de diferentes faculdades de Odontologia.

O referido questionário foi elaborado por Medeiros et al. ${ }^{1}$ (2007), indagando-se no referido formulário: "Quais os recursos complementares mais utilizados na detecção da sensibilidade pulpar que você utiliza durante o exame do paciente?" Os dados foram reunidos e dispostos de modo a visualizar a questão formulada da seguinte forma:

1. Testes térmicos pelo frio ( )

1.1 - bastão de gelo ( )

1.2 - água fria ( ) 
1.3 - algodão embebido em álcool ( )

1.4 - jato de ar ( )

1.5 - gases refrigerantes ( )

1.5.1 - cloreto de etila ( )

1.5.2 - tetrafluoroetano ou butano/ propano ( )

1.5.3 - bastão de neve carbônica ( )

2. Testes térmicos pelo calor ( )

2.1 - bastão de guta-percha ( )

2.2 - brunidor aquecido ( )

2.3 - outros ( ) Citar

3. Teste elétrico pulpar ( )

4. Teste de cavidade ( )

5. Teste de anestesia ( )

6. Outros ( ) Citar

No próprio formulário investigou-se a especialidade, o ano de formatura do profissional e que o entrevistador não interferisse na resposta do consultado, a fim de se convalidar a veracidade das respostas durante o processo de coleta dos resultados.

Os profissionais poderiam, no questionário, fazer referência a um ou mais recursos auxiliares, segundo suas necessidades clínicas.

De posse dos resultados obtidos, valeuse de estatística descritiva, utilizando tabela e dados relativos. Como inferência estatística foi utilizado teste binomial para proporção, próprio para comparação de duas amostras independentes, sendo o nível de significância de 5\%.

\section{RESULTADOS}

Os resultados encontram-se expressos na Tabela 1.
Os dados foram reunidos de modo a facilitar a subdivisão dos testes em frio, calor, elétrico, cavidade, anestesia e outros testes, fato este que permitiu estabelecer os percentuais de utilização em função do número de citações. Todos os 106 entrevistados apontaram mais de uma resposta, às vezes cinco testes relacionados no questionário.

Na comparação entre a proporção de clínicos gerais que utilizam guta-percha $(90,56 \%)$ e bastão de gelo $(77,35 \%)$, o teste apresentou pvalor $<0,05$, mostrando que a proporção daqueles que utilizam gutapercha é maior do que daqueles clínicos que usam bastão de gelo no dia-a-dia de suas atividades clínicas para determinar a condição pulpar do dente em questão.

\section{DISCUSSÃO}

Os recursos auxiliares ou complementares em endodontia são de importância fundamental na determinação do diagnóstico do estado da polpa dentária, inclusive no que respeita ao diagnóstico diferencial para avaliar a condição deste tecido.

Além do mais, a utilização desses recursos deve facilitar a identificação do ponto de vista clínico do estado pulpar, suficiente para detectar, sobretudo, a presença de tecido ou capaz de estimular prontamente a unidade sensorial pulpar.

Isso posto, aconselha-se, dentre os procedimentos semiotécnicos à disposição do clínico, a utilização do resfriamento ou aquecimento, que deverão ser aplicados na superfície externa do dente, porquanto, o tecido pulpar encontra-se enclausurado

Tabela 1 - Frequência, em números e percentagens, dos recursos usados de acordo com a escolha dos clínicos gerais.

\begin{tabular}{cccc} 
Teste & Produto & No de citações $^{0}$ \% Do total \\
& Bastão de gelo & 82 & 77,35 \\
Frio & Água fria & 26 & 24,52 \\
& Algodão em álcool & 1 & 0,94 \\
& Jato de ar & 44 & 41,50 \\
Calor & Gás refrigerante & 68 & 64,15 \\
Calor & Bastão de guta-percha aquecida & 96 & 90,56 \\
Elétrico & Brunidor aquecido & 5 & 4,71 \\
Cavidade & Pulp test & 2 & 1,88 \\
Anestesia & Broca & 33 & 31,13 \\
\hline \hline
\end{tabular}

MEDEIROS JMF

PINTO CA

ROSA LCL

HABITANTE SM

ALMEIDA ETDC

ZOLLNER NA

AVALIAÇÃO DA

ESCOLHA DOS

TESTES DE

SENSIBILIDADE

PULPAR POR

CLÍNICOS GERAIS

DA CIDADE DE

TAUBATÉ

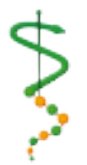

REVISTA DE

ODONTOLOGIA DA

UN I VERS I DADE

Cidade de São

PAULO

$2010 ; 22(1): 30-8$ JAN - ABR 
MEDEIROS JMF

PINTO CA

ROSA LCL

HABITANTE SM

ALMEIDA ETDC

ZOLLNER NA

AVALIAÇÃO DA

ESCOLHA DOS

TESTES DE

SENSIBILIDADE

PULPAR POR

CLINNICOS GERAIS

$D A$ CIDADE DE

TAUBATÉ

34

REVISTA DE

ODONTOLOGIA DA

UNIVERSIDADE

CIDADE DE SÃO

PAULO

$2010 ; 22(1): 30-8$,

$J A N-A B R$ na cavidade endodôntica, recoberto por esmalte e dentina, o que torna difícil sua inspeção.

Assim é que ressaltam os chamados testes de sensibilidade pulpar ou testes de vitalidade pulpar também conhecidos como testes térmicos. O objetivo de sua aplicação é promover significativas mudanças de temperatura na superfície do dente, objetivando uma resposta dolorosa.

Aliás, o diagnóstico é de fundamental importância na especialidade endodôntica para a determinação do tratamento a ser realizado, como uma arte em identificar uma doença a partir dos seus sinais e sintomas, já que é baseado essencialmente na interpretação ponderada dos dados obtidos durante o exame do paciente.

Desse modo, dominar a arte do diagnóstico significa aprender os princípios da coleta e interpretação de dados, registrando sistematicamente os sinais e sintomas por meio de análise minuciosa destes e estabelecer um correto diagnóstico levando a uma condução adequada do tratamento.

Paiva e Antoniazzi ${ }^{22}$ (1991) comentam que não há um procedimento ideal para todas as situações clínicas, todavia, há que se considerar que o conjunto de manobras empregadas na identificação da sintomatologia atual deve ser criterioso e significativo na medida em que se harmoniza com a queixa principal, história pregressa e atual bem como o exame físico e por fim o exame radiográfico.

A intenção desta pesquisa é situar quais os métodos empregados pelos clínicos gerais da cidade de Taubaté nessa etapa essencial da terapia endodôntica, quando se faz a determinação do diagnóstico.

Ocorrência de grande valor foi observada nesta investigação, ou seja, dos 106 registros coletados, 76 apontavam uso de testes procedentes do final do século XIX e começo do século passado, como, por exemplo, a água fria, algodão embebido em álcool, jato de ar e brunidor aquecido, fato este observado também por Medeiros et al. ${ }^{1}$ (2007) com 63 escolhas dos 200 questionários apresentados por especialistas em endodontia. Assim é que, graças ao desconhecimento científico e dos novos rumos que tomaram o diagnóstico, esses testes continuam ainda a ser empregados. A evolução dos testes na determinação da vitalidade ou sensibilidade pulpar foi legada ao segundo plano, sendo que a água fria já utilizada a diferentes temperaturas por Jack ${ }^{3}$ (1899) foi escolhida por 26 entrevistados, fato este também constatado por Medeiros et al. ${ }^{1}$ (2007) com 25 especialistas.

Nesse particular, muitos clínicos gerais ,por impulso, seguram a seringa e acionam jato de água no dente para confirmar as informações apresentadas pelo paciente no interrogatório dirigido. Portanto, tratase de uma atitude imediata do profissional, que utiliza o recurso mais próximo, ou seja, o jato de água fria aplicada por meio da seringa diretamente ao dente suspeito a fim de certificar-se da informação prestada pelo paciente. Afinal, isso não representa um erro na escolha do teste, já que o paciente afirma que seu dente dói com água fria. Evidente que se desconhece a princípio qual a temperatura empregada da água e o real efeito deste pouco resfriamento sobre a resposta pulpar.

Quanto ao algodão embebido em álcool escolhido por apenas um profissional $(0,94 \%)$ neste estudo em comparado com Medeiros et al. ${ }^{1}$ (2007) em 4 escolhas por especialistas $(2 \%)$, desconhece-se o efeito que a desidratação pode determinar na estrutura dentária e a possibilidade de promover sensibilidade dolorosa. Com relação ao brunidor aquecido, é desconhecido o grau de aquecimento do metal e os efeitos deletérios deste quando aplicado à superfície e à polpa dentária e, no entanto, 5 profissionais o elegeram $(4,71 \%)$ contra 9 especialistas (4,5\%) que escolheram esse teste (Medeiros et al. ${ }^{1}$ 2007). Embora esses testes sejam de fácil aplicação, podem ser utilizados, mas sempre com muita prudência, a despeito de suas limitações.

Relativamente ao teste de cavidade citado 33 vezes por clínicos gerais como recurso suplementar de exame, pode-se considerar o uso desse teste como definitivo e de grande confiabilidade na avaliação da sensibilidade pulpar, mesmo que sua utilização seja, em geral, imprudente, pois, representa um recurso invasivo, devendo ser usado em ocasiões exclusivas e quando não se concluiu sobre a vitalidade 
ou não do dente testado, evitando o seu emprego rotineiro. Quando confrontados os resultados da escolha desse teste no presente estudo com aqueles providenciados por Medeiros et al. ${ }^{1}$ (2007), observouse praticamente concordância de resultados do ponto de vista numérico, isto é, 33 citações contra 31 escolhas para os especialistas em Endodontia.

Outro percentual $(38,67)$ encontrado na escolha dos recursos foi o teste de anestesia por 41 profissionais, achados estes contrastantes $(5,0 \%)$ em relação àqueles dados encontrados por Medeiros et al. ${ }^{1}$ (2007). Essas referências se devem às circunstâncias de dores reflexas, uma vez que o diagnóstico diferencial, em caso de sinalgias, é facilitado pelo uso da anestesia (Lage-Marques e Antoniazzi ${ }^{21}$ 2002). Há uma grande dificuldade em se situar o diagnóstico correto dessas sinalgias dento-dentais e dento-faciais. Acredita-se que o profissional, seja especialista ou não em algumas situações, não consegue identificar o dente pulpítico.

Observou-se que apenas 2 profissionais elegeram o teste elétrico como recurso auxiliar de exame, talvez por não saberem estes dois entrevistados da ocorrência de falsos resultados positivos ou negativos que esse método proporciona, fato este não verificado por Medeiros et al. ${ }^{1}$ (2007) quando entrevistaram 200 especialistas em Endodontia. Aliás, talvez esse ponto que não foi comum entre os achados deste estudo com aqueles providenciados por Medeiros et al. ${ }^{1}$ (2007) seja devido ao arsenal de recursos que o especialista tem em mãos, enquanto o clínico geral é mais propenso ao uso de métodos mais tradicionais de exame. Além disso, o teste eléctrico é um método suplementar de boa aplicabilidade clínica, sobretudo, os de uso mais recente, embora esse teste ofereça menor confiabilidade do que os gases refrigerantes (Aun et al. ${ }^{7}$ 1992, Aun et al. ${ }^{8}$ 1994).

Como era de se esperar, a resposta à pergunta colocada neste questionário reverteu-se invariavelmente na escolha do emprego de recursos de fácil manipulação e aplicação, de vantagens reconhecidamente observadas na prática clínica e de eficácia clínica comprovada.
Causou surpresa o elevado índice de citações $(90,56 \%)$ da guta-percha aquecida, sendo a primeira colocada, representando 96 citações dos 106 entrevistados, talvez por se tratar de recurso universalmente aceito. A bem da verdade, o teste de guta-percha aquecido constitui método fácil de ser executado e de baixo custo e por essa razão foi eleito pelos entrevistados. Na falta do bastão de gelo, optou-se pelo bastão de guta-percha, entendendose que a falta de uma geladeira no consultório, representando equipamento de alto custo, é um bom motivo para escolha de um bastão de guta-percha de baixo valor aquisitivo.

Seguiu-se, na preferência dos clínicos gerais, o bastão de gelo em 77,35\%, o que não causou surpresa, pois o referido recurso foi o segundo colocado por especialistas em Endodontia na cidade de São Paulo, ou seja, 119 citações (59,5\%) do total de entrevistados, estudo este realizado pelos autores retrocitados (Aun et al. ${ }^{7}$ 1992).

O gás refrigerante não foi citado em 38 questionários como recurso complementar de exame, isto é, em 35,85\% dos clínicos gerais entrevistados. Acredita-se que tal ocorrência se deve às pesquisas em torno de sua utilização já há algum tempo. Desde sua introdução no mercado odontológico, é cada vez mais crescente a busca desta substância refrigerante mesmo pelos clínicos gerais da cidade de Taubaté, que, sem sombra de dúvida, sofrem influência da Universidade de Taubaté, que tem criado uma filosofia de pensamento em torno do uso dessa substância.

Então, dos 106 entrevistados, cerca de 68 clínicos gerais $(64,15 \%)$ referendaram o gás refrigerante, sendo que, em 3 casos, foi escolhido o cloreto de etila; em 5 situações, o bastão de neve carbônica e 60 casos os clínicos gerais elegeram o propano/ butano ou o tetrafluoroetano.

Aliás, diga-se a propósito que, os gases refrigerantes representam recurso de grande aceitação não só pelos especialistas em Endodontia, como também de outras áreas 7, 8, 14, 1, 13, 11, 9, 6 .

É importante salientar as observações de Zöllner e Pesce ${ }^{23}$ (1997), ao pesquisarem sobre a eficácia dos agentes térmicos
MEDEIROS JMF

PINTO CA

ROSA LCL

HABITANTE SM

ALMEIDA ETDC

ZOLLNER NA

AVALIAÇÃO DA

ESCOLHA DOS

TESTES DE

SENSIBILIDADE

PULPAR POR

CLÍNICOS GERAIS

DA CIDADE DE

TAUBATÉ
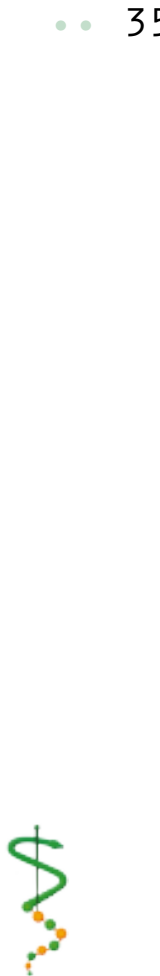

Revista de

ODONTOLOGIA DA

Universidade

CIDADE DE SÃO

PAULO

2010; 22(1):30-8 JAN-ABR 
MEDEIROS JMF

PINTO CA

ROSA LCL

HABITANTE SM

ALMEIDA ETDC

ZOLLNER NA

AVALIAÇÃO DA

ESCOLHA DOS

TESTES DE

SENSIBILIDADE

PULPAR POR

CLINICOS GERAIS

DA CIDADE DE

TAUBATÉ

36 na determinação da vitalidade pulpar. Os aludidos autores concluíram que o teste térmico, valendo-se da guta-percha aquecida, não parece uma boa opção de escolha quando comparado com outros testes de vitalidade pelo frio, a exemplo dos gases refrigerantes, considerados os mais eficazes de todos os testes de vitalidade o que, aliás, contraria os achados de escolha de testes diagnósticos no presente trabalho.

Finalizando, convém reafirmar as considerações do relato de Nicholls ${ }^{20}$ (1984) sobre a escolha indiscriminada de vários testes durante o exame do paciente, justificando que alguns profissionais utilizam um único teste de sensibilidade pulpar enquanto outros utilizam vários testes, o que significa que, em determinados casos, o diagnóstico é tão difícil que sua resolução necessita de vários métodos de exame. Em outras condições, a aplicação de apenas um recurso complementar provoca uma resposta tão explícita que, por si só, apresenta a identificação do problema.

\section{CONCLUSÕES}

Perante a metodologia empregada, parece lícito afirmar que, dentre os métodos suplementares de exame do paciente relacionados no formulário, os mais usados na determinação da sensibilidade pulpar foram, em ordem crescente: algodão embebido em álcool (1), teste elétrico (2), brunidor aquecido (5), água fria (26), teste de cavidade (33), teste de anestesia (41), jato de ar (44), gás refrigerante (68), bastão de gelo (82) e guta-percha aquecida (96). 
1. Medeiros JMF, Carvalho PL, Alkmin ST, Zöllner NA, Haddad Filho MS. Avaliação da escolha dos testes de sensibilidade pulpar por especialistas em endodontia. Rev Portuguesa de Estomatol, Med Dent e Cirurg Maxilofacial 2007 48(3): 149-154.

2. Castagnola L, Negro V. L'esame delle vitalita pulpare nella pratica. Mondo Odontostomatol 1972 nov-dez; 14(6): 919-31.

3. Jack L. Observation of the relation of thermal irritation of the teeth to their treatment. Dental Cosmos 1899; 41(1):1-6.

4. Austin LT, Waggener DT. Vitality tests with particular reference to the use of ice. J Am Dent Assoc 1941 Jul; 28(7): 1044-49.

5. Dachi SF, Haley JV, Sanders JE. Standardization of a test for dental sensivity to cold. Oral Surg Oral Med Oral Pathol 1967 Nov; 24(5): 687-92.

6. Pesce HF, Medeiros JMF, Rizzo VA. Determinação da vitalidade pulpar pelo teste térmico do frio. Rev Paul Odontol 1985 set-out; 7(5): p.2-10.

7. Aun CE, Caldeira CL, Gavini G, Pesce HF. Avaliação da vitalidade pulpar em dentes permanentes jovens com rizogênese completa. Rev Fac Odontol FZL 1992 jul-dez; 4(2): 95-104.

8. Aun CE, Caldeira CL, Gavini G, Pesce HF. Avaliação da vitalidade pulpar em dentes permanentes jovens com rizogênese incompleta. Rev Paul Odontol 1994 novdez;16(6): 9-16.

9. Pesce HF, Carrascoza A, Medeiros JMF. Determinação da vitalidade pulpar em dentes portadores de bandas ortodônticas. Rev Pós-Grad Fac Odontol Univ São Paulo 1997; 4(2):93-7.

10. Medeiros JMF, Pesce HF. Eficácia do bastão de gelo e tetrafluoroetano na determinação da vitalidade pulpar. Rev Odontol Univ São Paulo 1997 jul-set; 11(3): 215-9.

11. Medeiros JMF, Pesce HF. Confiabilidade do gelo e tetrafluoroetano na determinação da vitalidade pulpar. Rev Odontol Univ São Paulo 1998 jan-mar; 12(1): 19-27.

12. Medeiros JMF, Caldeira CL, Haddad Filho MS, Machado MEL Eficácia de dois agentes térmicos em dentes com coroa protética RGO (Porto Alegre) 2004 jul-set; 52(3): 197-200.

13. Medeiros JMF, Machado MEL, Caldeira CL, Zöllner NA, Haddad Filho MS, Gavini G. Eficácia de dois agentes térmicos antes e após o tratamento ortodôntico em dentes submetidos a procedimentos restauradores. Publ UEPG Biol Healt As (Pontagrossa) 2005 jun; 11(2): 27-34.

14. Haddad Filho MS, Caldeira CL, Medeiros JMF. Confiabilidade do gelo e tetrafluoroetano em dentes com pulpite irreversível. Rev Assoc Bras Odontol 2009; 17(3):165171.

15. Irala LED. Avaliação comparativa, in vitro, da capacidade de abaixamento da temperatura de diferentes agentes refrigerantes em sua fonte e meios de transporte. [Mestrado] - Rio Grande do Sul - CO/ULBRA, 2003.

16. Pesce HF, Barletta FB, Medeiros, JMF, Machado MEL. An in vitro evaluation of the effects of three thermal pulp testing methods on intrapulpal temperature. Rev Odontol UNICID 1995 jan-jun; 7(1): 7-11.

17. Pesce HF, Medeiros JMF. Determinação da vitalidade pulpar. In: Maciel RN Oclusão e ATM: procedimentos clínicos. 1 ed. São Paulo: Santos, 1998.107-110.

PINTO CA

ROSA LCL

HABITANTE SM

ALMEIDA ETDC

ZOLLNER NA

AVALIAÇÃO DA

ESCOLHA DOS

TESTES DE

SENSI BILIDADE

PULPAR POR

CLÍNICOS GERAIS

DA CIDADE DE

tAUBATÉ

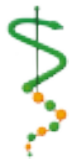

REVISTA DE

ODONTOLOGIA DA

UN I VERS I DADE

Cidade de são

PAULO

$2010 ; 22(1): 30-8$

JAN - ABR 
MEDEIROS JMF

PINTO CA

ROSA LCL

HABITANTE SM

ALMEIDA ETDC

ZOLLNER NA

AVALIAÇÃO DA

ESCOLHA DOS

TESTES DE

SENSIBILIDADE

PULPAR POR CLINICOS GERAIS

$D A$ CIDADE DE

TAUBATÉ

18. Barletta FB. Avaliação "in vitro" dos possíveis efeitos danosos na superfície do esmalte dentário humano frente ao emprego do bastão de neve carbônica. [Doutorado] - São Paulo: Faculdade de Odontologia da Universidade de São Paulo. 1994.

19. Cohen S, Burns RC. Caminhos da polpa. 7 ed. Rio de Janeiro: Guanabara-Koogan, 2000. p.1-18

20. Nicholls E. Endodontics. 3th ed. London: Bristol, 1984. v.11, p.18-20

21. Lage-Marques JLS, Antoniazzi JH. Técnica endodôntica. Versão Eletrônica da Técnica de Endodontia da Faculdade de Odontologia da Universidade de São Paulo. [CD-ROM]. São Paulo: Ajna Interactive; 2002.

22. Paiva JG, Antoniazzi JH. Endodontia. Bases para a prática clínica. 2. ed. São Paulo: Artes Médicas, 1991. 886p.

23. Zöllner NA, Pesce HF. Considerações em torno do emprego dos testes térmicos na determinação da vitalidade pulpar. Rev Biociências 1997; 3:113-20.

Recebido em: 05/03/2010

Aceito em: 16/03/2010 